\title{
Prognostic role of miR-760 in hepatocellular carcinoma
}

\author{
DONG SUN ${ }^{1,2}$, JINGHUI LU $^{1}$, CHUNXIAO HU ${ }^{1}$, QIANGBO ZHANG ${ }^{1}$, \\ XIANGDONG WANG ${ }^{2}$, ZONGLI ZHANG ${ }^{1}$ and SANYUAN HU ${ }^{1}$ \\ ${ }^{1}$ Department of General Surgery and ${ }^{2}$ School of Basic Medical Sciences, \\ Qilu Hospital of Shandong University, Jinan, Shandong 250012, P.R. China
}

Received March 7, 2018; Accepted September 26, 2018

DOI: $10.3892 / \mathrm{ol} .2018 .9546$

\begin{abstract}
Previous studies have demonstrated that microRNA (miR)-760 serves an important role in various cancer types. However, to the best of our knowledge, its role in hepatocellular carcinoma (HCC) has not been fully elucidated. The current study investigated the prognostic role of miR-760 in HCC by using the Kaplan-Meier plotter database. The current data indicated that low expression of miR-760 was associated with higher overall survival (OS) for all patients with HCC from both the RNA-seq [hazard ratio $(\mathrm{HR})=2.04 ; 95 \%$ confidence interval $\left.(\mathrm{CI})=1.44-2.89 ; \mathrm{P}=4.9 \times 10^{-5}\right]$ and the non-commercial spotted microarray $(\mathrm{HR}=1.71 ; \mathrm{CI}=1.05-2.76 ; \mathrm{P}=0.028)$. In the RNA-seq platform, a lower expression of miR-760 was strongly associated with improved OS in male patients with HCC, but not in female patients with HCC. Additionally, low expression of miR-760 was associated with improved OS in patients with stage I, II and III HCC, and was associated with improved OS in Asian and Caucasian patients. The current results indicated that miR-760 serves as an oncogene for HCC and high expression of miR-760 is significantly associated with tumor progression and poor prognosis in patients with HCC.
\end{abstract}

\section{Introduction}

Hepatocellular carcinoma (HCC), the most common type of liver cancer in adults, occurs more frequently in males compared with females (1). The incidence of HCC is

Correspondence to: Dr Sanyuan $\mathrm{Hu}$ or Dr Zongli Zhang, Department of General Surgery, Qilu Hospital of Shandong University, 107 Wenhua Road, Jinan, Shandong 250012, P.R. China E-mail:22993681@qq.com

E-mail: 1442776131@qq.com

Abbreviations: HCC, hepatocellular carcinoma; HR, hazard ratio; KM, Kaplan-Meier; OS, overall survival; miR, microRNA; OSF, oral submucous fibrosis; GC, gastric cancer; CRC, colorectal cancer; CC, colon cancer; OC, ovarian cancer; PHLPP2, PH domain and leucine rich repeat protein phosphatase 2; NSCLC, non-small cell lung cancer

Key words: microRNA-760, hepatocellular carcinoma, Kaplan-Meier plotter, prognostic role increasing worldwide due to an increased rate of hepatitis virus infection (1). Chronic liver inflammation, due to hepatitis infection or exposure to toxins, is thought to be the leading cause of $\mathrm{HCC}(2,3)$. Many previous studies suggest that microRNAs (miRNAs/miRs), small non-coding RNA molecules, 22 nucleotides in length (4), serve an essential role in the progression of HCC (4-7). Notably, recent studies of serum from patients with liver diseases have indicated that circulating miRNAs are a novel class of biomarkers of HCC and other liver diseases $(6,7)$. These studies suggest serum miRNAs may be potential biomarkers for HCC. Therefore, it is critical to identify novel miRNA biomarkers to help predict the progression of $\mathrm{HCC}$.

Human miR-760 is involved in the pathogenesis of various human diseases and its expression varies dramatically in numerous pathological conditions. In patients with oral submucous fibrosis (OSF) the expression of miR-760 is decreased in OSF tissue compared with normal tissue (8). In $\mathrm{T}$ cells of patients with rheumatoid arthritis the expression of miR-760 is increased compared with that in T cells from healthy individuals (9). In addition, miR-760 has been identified as a novel biomarker to predict the progression of various cancer types, including gastric cancer (GC) (10), breast cancer (11-13), colorectal cancer (CRC) (14), colon cancer (CC) and ovarian cancer (OC) (15). Based on an RNA-seq analysis of bone marrow cells from patients with GC, the expression of miR-760 in bone marrow and tumor tissue is significantly lower in stage IV patients in comparison with stage I patients (10). A higher miR-760 expression was also associated with better overall survival (OS) in patients with GC (10). This may be due to the direct interaction of miR-760 with histone mRNA, resulting in the decreased expression of histone cluster $1 \mathrm{H} 3$ family member D (10). Furthermore, an in vitro study indicated that miR-760 regulates breast cancer stem cell metastasis and gene expression by targeting Nanog homeobox dependent pathways (16). miR-760 is also significantly downregulated in breast cancer cells that exhibit chemoresistance (12). By contrast, overexpression of miR-760 induces the resistance of human breast cancer cells to doxorubicin (11). In human CC cells and replicatively senescent human lung fibroblast cells, miR-760 expression is upregulated and regulates protein kinase CK2 $\alpha$ subunit expression (17). In Egyptian patients with $\mathrm{CRC}$, miR-760 is significantly downregulated in CRC tissue compared with healthy tissue and the expression of miR-760 is inversely associated with 
advanced CRC stages (18). Previously, miR-760 has been detected in the plasma of patients with CRC and further investigation demonstrated that plasma miR-760 may be a potential novel biomarker for the early stages of CRC (14). A systematic review and meta-analysis in gastrointestinal cancer types has also indicated that miR-760 is downregulated and associated with poor prognosis (19). By contrast, a quantitative polymerase chain reaction assay for miR-760 in OC tissues revealed a markedly higher expression of miR-760 in tumor tissue compared with healthy tissue. Additionally, this assay demonstrated that miR-760 directly regulates the expression of $\mathrm{PH}$ domain and leucine rich repeat protein phosphatase 2 (PHLPP2), which subsequently results in tumor cell proliferation (15). The low expression of miR-760 is strongly associated with a better phenotype and prognosis for patients with OC, suggesting that miR-760 serves an oncogenic role in human OC progression (15). However, to the best of our knowledge, the role of miR-760 in patients with HCC has not been studied.

The Kaplan-Meier (KM) plotter miRpower analysis tool has been reported to analyze the prognostic role of miRNAs in breast cancer (20). The current study used the KM plotter database to investigate the prognostic role of miR-760 expression in HCC patients.

\section{Materials and methods}

To analyze the prognostic role of miRNA expression in HCC, the current study used the KM plotter database and miRpower analysis tool to study the association of miR-760 expression with OS in different analysis selections (http://kmplot. com/analysis/index.php? $\mathrm{p}=$ service\&cancer=liver_mirna). The KM plotter database has combined the published miRNA expression, OS and clinical data from The Cancer Genome Atlas (http://cancergenome.nih.gov), Gene Expression Omnibus (http://www.ncbi.nlm.nih.gov/geo/), European Genome-Phenome Archive (https://www.ebi.ac.uk/ega/home) and PubMed (http://www.pubmed.com) (21). The database included the miRNA expression and OS rates (with a 10 year follow-up) of 614 patients with HCC.

The KM plotter analysis tool was used to obtain Kaplan-Meier plots for the OS rate of patients with HCC. hsa-miR-760 was entered into the database and the number-at-risk was displayed under the main panel of the OS. Based on the expression of miR-760, patients were divided into two groups. Patients with miR-760 expression higher than the median were pooled into the group with high expression, while the patients with miR-760 expression lower than the median were pooled into the group with low expression. Other statistical outcomes calculated from the database, including hazard ratio (HR), 95\% confidence intervals and log rank P-values, were also included in the figures and table in the current study. $\mathrm{P}<0.05$ was considered to indicate a statistically significant difference.

\section{Results}

The current study used the KM plotter database and analysis tool to determine the prognostic value of miR-760 for HCC. Survival curves were generated for patients with HCC from the RNA-seq platform ( $\mathrm{n}=372$; Fig. 1A) and from the non-commercial spotted microarray (n=164; Fig. 1B). Compared with high expression, low expression of miR-760 was associated with higher OS for all patients with HCC from both platforms (Fig. 1). In the RNA-seq platform, the HR for miR-760 was 2.04 [95\% confidence interval $(\mathrm{CI})=1.44-2.89$ ] and $\mathrm{P}=4.9 \times 10^{-5}$ (Fig. 1A), and in the non-commercial spotted platform the HR for miR-760 was $1.71(95 \% \mathrm{CI}=1.05-2.76)$ and $\mathrm{P}=0.028$ (Fig. 1B).

Subsequently, the current study analyzed the expression of miR-760 in patients with HCC of different sex (Table I and Fig. 2), different clinical stages (Table I and Fig. 3) and different ethnicities (Table I and Fig. 4) using the data from the RNA-Seq platform. As demonstrated in Fig. 2 and Table I, compared with high miR-760 expression, low expression of miR-760 was associated with a higher OS in male patients with HCC [hazard ratio $(\mathrm{HR})=2.36(1.51-3.67)$, $\mathrm{P}=9.2 \times 10^{-5}$; Fig. $\left.2 \mathrm{~A}\right]$, but not in female patients with HCC [HR=1.58 (CI=0.89-2.82), P=0.11; Fig. 2B]. As demonstrated in Fig. 3 and Table I, compared with high miR-760 expression, low expression of miR-760 was associated with a higher OS in stage I patients $[\mathrm{HR}=2.19(\mathrm{CI}=1.18-4.05), \mathrm{P}=0.01$; Fig. 3A], stage II patients [HR=3.44 ( $\mathrm{CI}=1.49-7.93), \mathrm{P}=0.002$; Fig. $3 \mathrm{~B}]$ and stage III patients $[\mathrm{HR}=2.29(\mathrm{CI}=1.18-4.44)$, $\mathrm{P}=0.01 ;$ Fig. 3C]. Stage IV patients was excluded due to a low population. Additionally, compared with high expression, low miR-760 expression was associated with a higher OS in Asian patients and Caucasian patients (Fig. 4 and Table I). As demonstrated in Fig. 4 and Table I, in the 393 patients with complete ethnicity information (29 patients were excluded in this study due to the missing of ethnicity information), the patients with low miR-760 expression was marginally associated with higher OS in Caucasian patients [HR=1.99 (CI-1.05-3.8), P=0.032; Fig. 4A] and was strongly associated with higher $\mathrm{OS}$ in Asian patients [HR=3.44 (CI=1.82-6.51), $\mathrm{P}=5.3 \times 10^{-5}$; Fig. 4B].

\section{Discussion}

As the primary cancer of the liver, HCC is one of the most prevalent cancer types and affects more than $5 \times 10^{5}$ individuals globally every year (22). HCC is associated with a poor prognosis rate; the five-year survival rate of patients with $<10 \%$ (23). This may be due to various risk factors and limited approaches for early diagnosis $(22,23)$. Liver transplantation and resection are commonly used as the most effective clinical therapies for HCC worldwide (24). However, the worldwide incidence rate of $\mathrm{HCC}$ and $\mathrm{HCC}$-associated cases of mortality are very high, particularly in developing countries, including China and countries in Africa (2). Previously, it has been identified that various protein-coding genes serve indispensable roles in the onset and development of HCC in humans (25). HCC is commonly not detected until it is in the advanced stage $(2,23,24)$, therefore identifying more sensitive biomarkers for early stage HCC may be beneficial for earlier diagnosis.

Recently, many fundamental and clinical studies have identified that miRNAs, including miR-21, $-122,-221 / 222,-520$ and -657 , are involved in the progression of HCC $(5,26-28)$. These studies may assist in the identification of novel biomarkers for early stage HCC and may also be beneficial in the development 
Table I. Association of miR-760 expression and patients with HCC with different clinical variables.

\begin{tabular}{|c|c|c|c|c|}
\hline Clinical variables & Cases, $\mathrm{n}$ & HR & $95 \% \mathrm{CI}$ & P-value \\
\hline \multicolumn{5}{|l|}{ Sex } \\
\hline Male & 253 & 2.36 & $1.51-3.67$ & $<0.001$ \\
\hline Female & 119 & 1.58 & $0.89-2.82$ & 0.115 \\
\hline \multicolumn{5}{|l|}{ Clinical stage } \\
\hline $\mathrm{I}$ & 172 & 2.19 & $1.18-4.05$ & 0.010 \\
\hline II & 86 & 3.44 & $1.49-7.93$ & 0.002 \\
\hline III & 85 & 2.29 & $1.18-4.44$ & 0.011 \\
\hline \multicolumn{5}{|l|}{ Ethnicity } \\
\hline Caucasian & 182 & 1.99 & $1.05-3.80$ & 0.032 \\
\hline Asian & 161 & 3.44 & $1.82-6.51$ & $<0.001$ \\
\hline
\end{tabular}

HR, hazard ratio; CI, confidence interval.
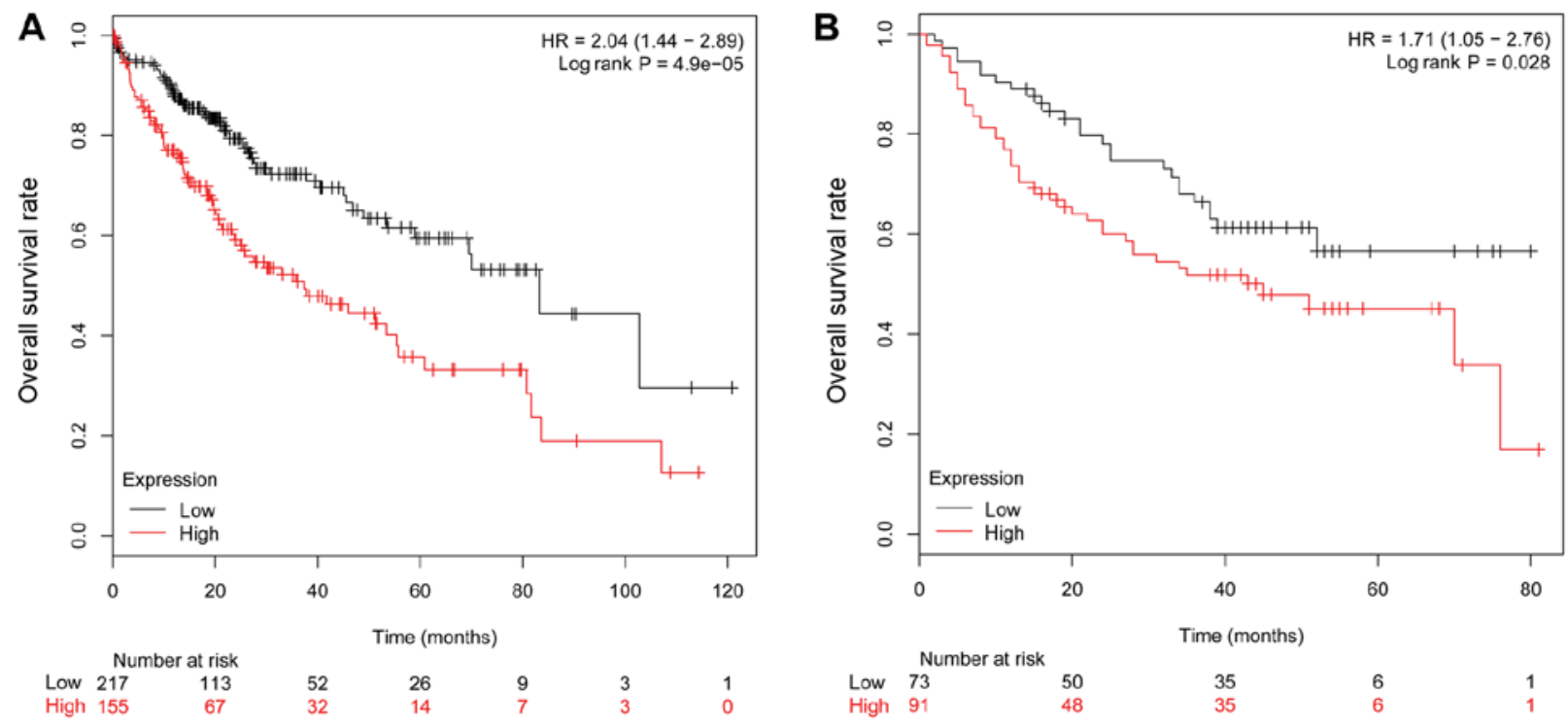

Figure 1. Prognostic value of miRNA-760 in patients with HCC. Survival curves are plotted for all patients with HCC in different platforms. (A) RNA-seq platform ( $n=372)$. (B) Non-commercial spotted platform $(n=164)$. HR, hazard ratio; HCC, hepatocellular carcinoma; miRNA, microRNA.

of precision therapy for patients with HCC. Notably, miRNAs have also been detected in the serum of patients with HCC $(29,30)$. Thus, monitoring serum miRNA profiles may be a convenient approach to identify early changes in the pathological functions of the liver during the onset and development of HCC.

The current study investigated the clinical value of miR-760 in predicting the development of HCC and the OS rate. The current data indicated that low expression of miR-760, compared with high expression, was associated with higher OS for patients with HCC and suggested that miR-760 may be a novel biomarker for HCC that serves as an oncogene in HCC development. In the development of non-small cell lung cancer (NSCLC), miR-760 suppresses the proliferation and metastasis of NSCLC by directly decreasing the expression of the proto-oncogene, tyrosine-protein kinase ROS (31). Furthermore, in human CRC, miR-760 attenuates tumor progression by targeting the basic leucine zipper transcriptional factor ATF-like 3 dependent signaling pathways (32). Notably, miR-760 has been demonstrated to serve different roles in the development of OC. The expression of miR-760 is increased in the tumor tissue of patients with OC compared with that in healthy tissue from patients with OC and miR-670 promotes proliferation of OC cells via the downregulation of PHLPP2 expression (15). Similar to OC development, miR-760 may negatively regulate the expression of cancer suppressing genes, including PHLPP2, to promote the progression of HCC. This potential function of miR-760 requires further investigation to provide a better understanding of the mechanistic details involved. The current detailed analysis of miR-760 expression in different sexes and ethnicities suggested that miR-760 would be more sensitive for predicting prognosis in male patients compared with female patients and more sensitive in Asian patients compared with Caucasian patients. Notably, 

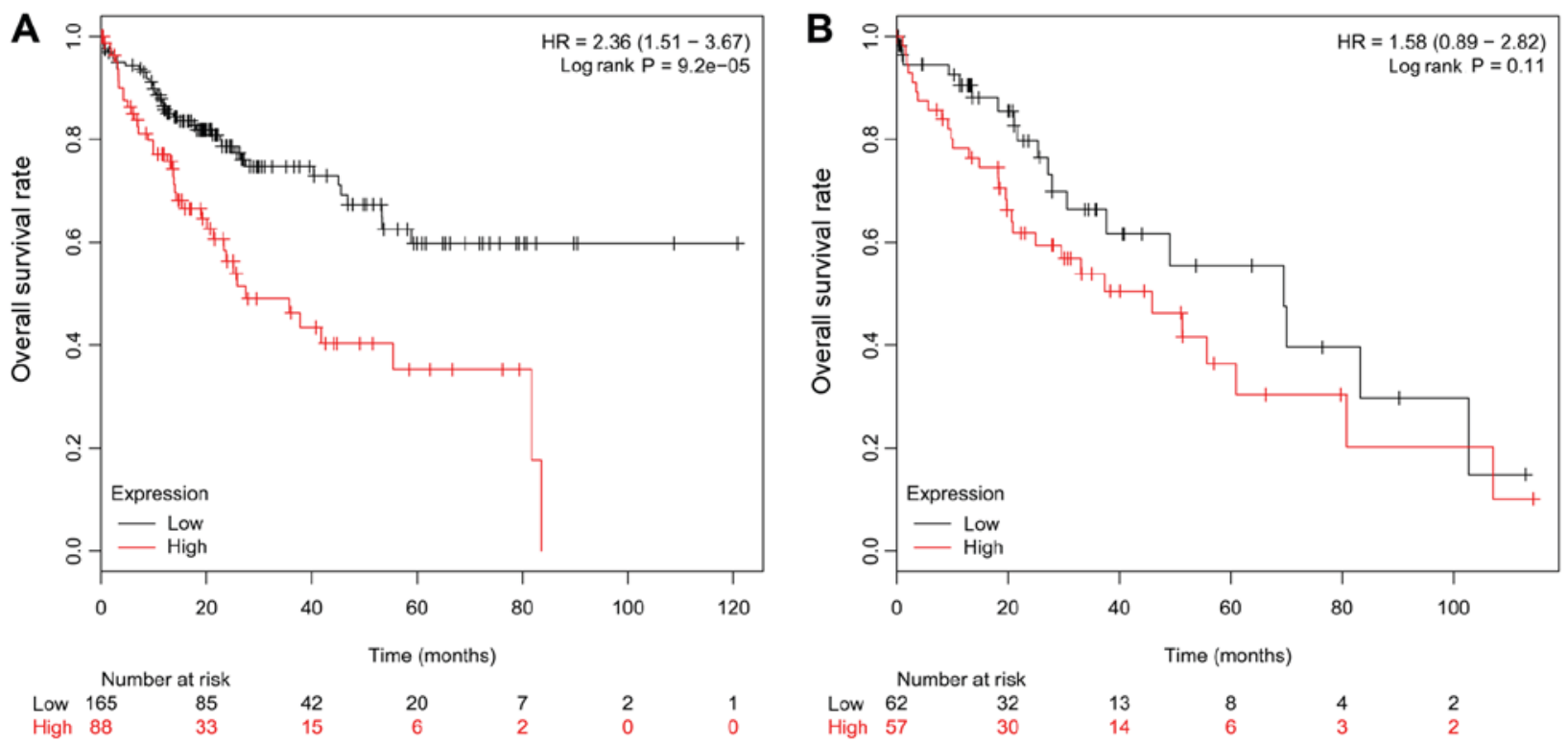

Figure 2. Prognostic value of miRNA-760 expression in patients with HCC of different sex. Survival curves are plotted for all patients with HCC of different sex. (A) Male (n=253). (B) Female (n=119). HR, hazard ratio; HCC, hepatocellular carcinoma; miRNA, microRNA.
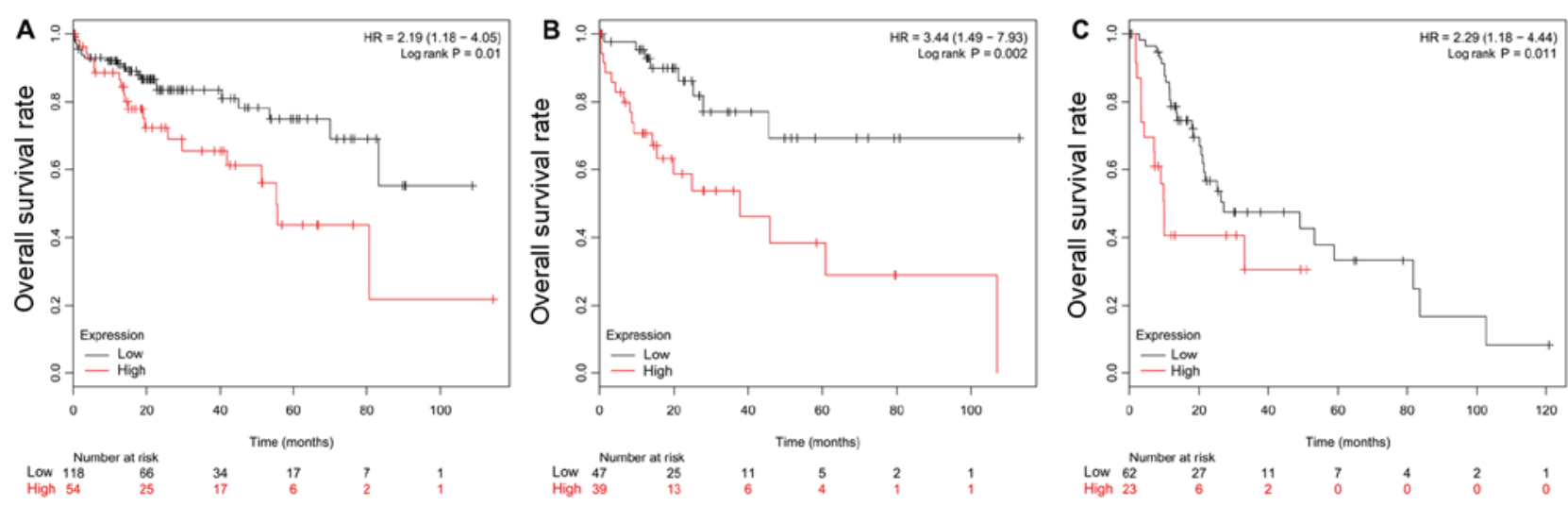

Figure 3. Prognostic value of miRNA-760 in patients with $\mathrm{HCC}$ at different stages. Survival curves are plotted for all patients with HCC at different stages. (A) Stage I (n=172). (B) Stage II ( $\mathrm{n}=86)$. (C) Stage III ( $\mathrm{n}=85)$. HR, hazard ratio; HCC, hepatocellular carcinoma; miRNA, microRNA.
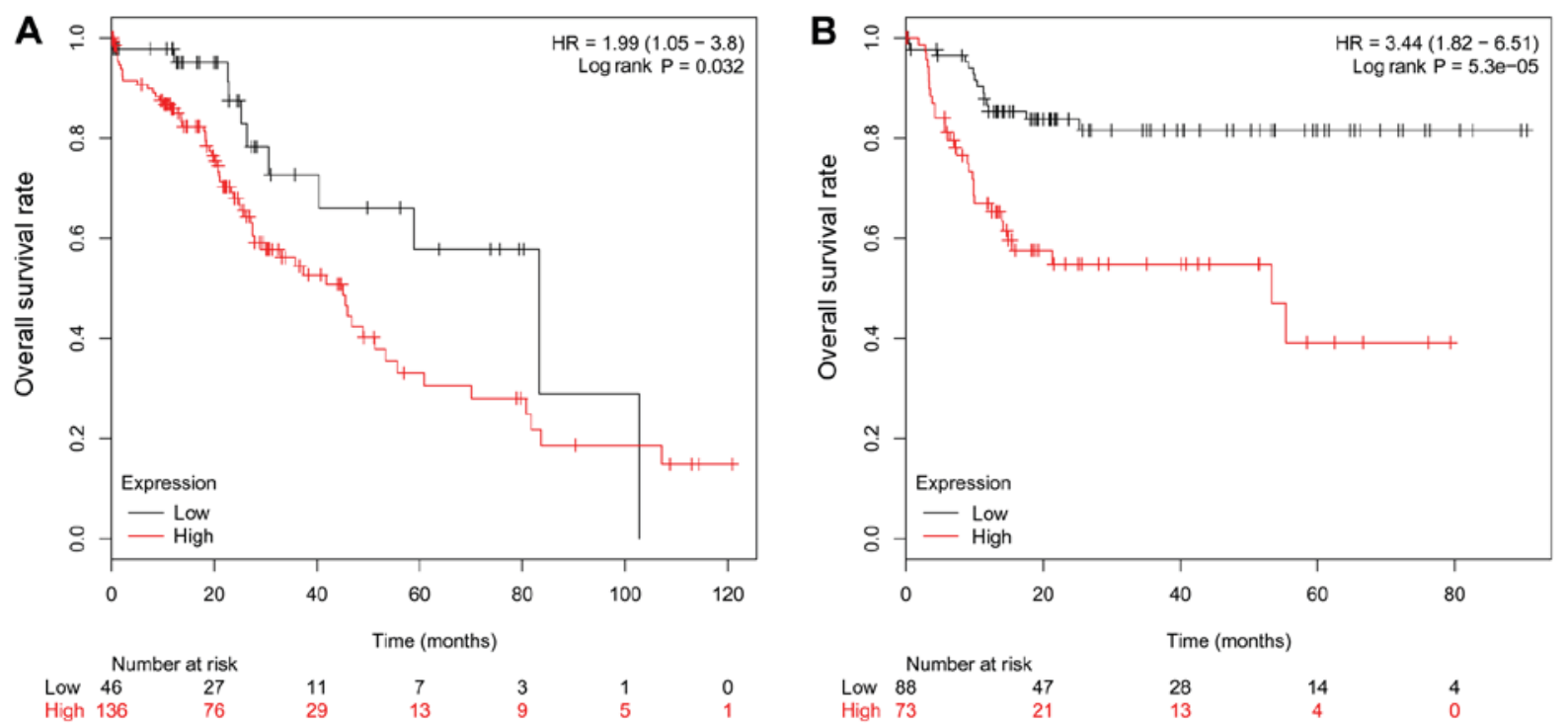

Figure 4. Prognostic value of miRNA-760 expression in patients with HCC of different ethnicity. Survival curves are plotted for patients with HCC of different ethnicity. (A) Caucasian patients $(\mathrm{n}=182)$. (B) Asian patients $(\mathrm{n}=161)$. HR, hazard ratio; HCC, hepatocellular carcinoma; miRNA, microRNA. 
compared with high miR-760 expression, low expression of miR-760 was strongly associated with a higher OS in patients with HCC in stage I, II and III.

In conclusion, the current study demonstrated the distinct prognostic role of miR-760 for HCC. Compared with high expression, low miR-760 expression was associated with better OS for all patients with HCC, particularly for male patients and Asian patients. Therefore, the current results suggest that miR-760 may be a novel biomarker for patients with HCC both in the early and advanced stages. Furthermore, by targeting miR-760, a novel approach for earlier diagnosis of HCC may be achieved, which is essential to improve the survival rate of patients with HCC.

\section{Acknowledgements}

Not applicable.

\section{Funding}

The present study was supported by the Key Research Project of Shandong Province (grant no. 2017GSF218087).

\section{Availability of data and materials}

All data generated or analyzed during the present study are included in this article.

\section{Authors' contributions}

DS, JL, CH, QZ, XW, ZZ and SH designed the experiments and collected the data; DS, JL, ZZ and SH contributed to the data analysis and wrote the manuscript.

\section{Ethics approval and consent to participate}

Not applicable.

\section{Patient consent for publication}

Not applicable.

\section{Competing interests}

The authors declare that they have no competing interests.

\section{References}

1. El-Serag HB: Epidemiology of viral hepatitis and hepatocellular carcinoma. Gastroenterology 142: 1264-1273 e1, 2012.

2. Gomaa AI, Khan SA, Toledano MB, Waked I and Taylor-Robinson SD: Hepatocellular carcinoma: Epidemiology, risk factors and pathogenesis. World J Gastroenterol 14: 4300-4308, 2008.

3. Rapisarda V, Loreto C, Malaguarnera M, Ardiri A, Proiti M, Rigano G, Frazzetto E, Ruggeri MI, Malaguarnera G, Bertino N, et al: Hepatocellular carcinoma and the risk of occupational exposure. World J Hepatol 8: 573-590, 2016.

4. Felekkis K, Touvana E, Stefanou CH and Deltas C: microRNAs: A newly described class of encoded molecules that play a role in health and disease. Hippokratia 14: 236-240, 2010.

5. Hung $\mathrm{CH}$, Chiu $\mathrm{YC}$, Chen $\mathrm{CH}$ and $\mathrm{Hu} \mathrm{TH}$ : MicroRNAs in hepatocellular carcinoma: Carcinogenesis, progression, and therapeutic target. Biomed Res Int 2014: 486407, 2014.
6. Ding Y, Yan JL, Fang AN, Zhou WF and Huang L: Circulating miRNAs as novel diagnostic biomarkers in hepatocellular carcinoma detection: A meta-analysis based on 24 articles. Oncotarget 8: 66402-66413, 2017.

7. Loosen SH, Schueller F, Trautwein C, Roy S and Roderburg C: Role of circulating microRNAs in liver diseases. World $\mathrm{J}$ Hepatol 9: 586-594, 2017.

8. Chickooree D, Zhu K, Ram V, Wu HJ, He ZJ and Zhang S: A preliminary microarray assay of the miRNA expression signatures in buccal mucosa of oral submucous fibrosis patients. J Oral Pathol Med 45: 691-697, 2016.

9. Lai NS, Yu HC, Tung CH, Huang KY, Huang HB and Lu MC: The role of aberrant expression of $\mathrm{T}$ cell miRNAs affected by TNF- $\alpha$ in the immunopathogenesis of rheumatoid arthritis. Arthritis Res Ther 19: 261, 2017.

10. Iwaya T, Fukagawa T, Suzuki Y, Takahashi Y, Sawada G, Ishibashi M, Kurashige J, Sudo T, Tanaka F, Shibata K, et al: Contrasting expression patterns of histone mRNA and microRNA 760 in patients with gastric cancer. Clin Cancer Res 19: 6438-6449, 2013.

11. Hu SH, Wang CH, Huang ZJ, Liu F, Xu CW, Li XL and Chen GQ: miR-760 mediates chemoresistance through inhibition of epithelial mesenchymal transition in breast cancer cells. Eur Rev Med Pharmacol Sci 20: 5002-5008, 2016.

12. Lv J, Fu Z, Shi M, Xia K, Ji C, Xu P, Lv M, Pan B, Dai L and Xie H: Systematic analysis of gene expression pattern in has-miR-760 overexpressed resistance of the MCF-7 human breast cancer cell to doxorubicin. Biomed Pharmacother 69: 162-169, 2015.

13. Lv J, Xia K and Xu P: miRNA expression patterns in chemoresistant breast cancer tissues. Biomedi Pharmacother 68: 935-942, 2014.

14. Wang Q, Huang Z, Ni S, Xiao X, Xu Q, Wang L, Huang D, Tan C, Sheng $\mathrm{W}$ and Du X: Plasma miR-601 and miR-760 are novel biomarkers for the early detection of colorectal cancer. PLoS One 7: e44398, 2012.

15. Liao Y, Deng Y, Liu J, Ye Z, You Z, Yao S and He S: MiR-760 overexpression promotes proliferation in ovarian cancer by downregulation of PHLPP2 expression. Gynecol Oncol 143: 655-663, 2016

16. Han ML, Wang F, Gu YT, Pei XH, Ge X, Guo GC, Li L, Duan X, Zhu MZ and Wang YM: MicroR-760 suppresses cancer stem cell subpopulation and breast cancer cell proliferation and metastasis: By down-regulating NANOG. Biomed Pharmacother 80: 304-310, 2016.

17. Lee YH, Kim SY and Bae YS: Upregulation of miR-760 and miR-186 is associated with replicative senescence in human lung fibroblast cells. Mol Cells: 620-627, 2014.

18. Elshafei A, Shaker O, Abd El-Motaal O and Salman T: The expression profiling of serum miR-92a, miR-375 and miR-760 in colorectal cancer: An Egyptian Study. Tumour Biol 39: $1010428317705765,2017$.

19. Zheng Q, Chen C, Guan H, Kang W and Yu C: Prognostic role of microRNAs in human gastrointestinal cancer: A systematic review and meta-analysis. Oncotarget 8: 46611-46623, 2017.

20. Lánczky A, Nagy Á, Bottai G, Munkácsy G, Szabó A, Santarpia L and Gyórffy B: miRpower: A web-tool to validate survival-associated miRNAs utilizing expression data from 2178 breast cancer patients 160: 439-446, 2016.

21. Győrffy B, Surowiak P, Budczies J and Lánczky A: Online survival analysis software to assess the prognostic value of biomarkers using transcriptomic data in non-small-cell lung cancer. PLoS One 8: e82241, 2013.

22. Ananthakrishnan A, Gogineni V and Saeian K: Epidemiology of primary and secondary liver cancers. Semin Intervent Radiol 23: 47-63, 2006.

23. Lee JG, Kang CM, Park JS, Kim KS, Yoon DS, Choi JS, Lee WJ and Kim BR: The actual five-year survival rate of hepatocellular carcinoma patients after curative resection. Yonsei Med J 47: 105-112, 2006

24. Waghray A, Murali AR and Menon KN: Hepatocellular carcinoma: From diagnosis to treatment. World J Hepatol 7: 1020-1029, 2015.

25. Wan DW, Tzimas D, Smith JA, Kim S, Araujo J, David R, Lobach I and Sarpel U: Risk factors for early-onset and late-onset hepatocellular carcinoma in Asian immigrants with hepatitis B in the United States. Am J Gastroenterol 106: 1994-2000, 2011.

26. Mehra $M$ and Chauhan R: Long noncoding RNAs as a key player in hepatocellular carcinoma. Biomark Cancer 9: 1179299X17737301, 2017. 
27. Gramantieri L, Fornari F, Callegari E, Sabbioni S, Lanza G, Croce CM, Bolondi L and Negrini M: MicroRNA involvement in hepatocellular carcinoma. J Cell Mol Med 12: 2189-2204, 2008.

28. Huang CS, Yu W,Cui H, Wang YJ, Zhang L, Han F and Huang T: Increased expression of miR-21 predicts poor prognosis in patients with hepatocellular carcinoma. Int J Clin Exp Pathol 8: 7234-7238, 2015.

29. Liu AM, Yao TJ, Wang W, Wong KF, Lee NP, Fan ST, Poon RT, Gao C and Luk JM: Circulating miR-15b and miR-130b in serum as potential markers for detecting hepatocellular carcinoma: A retrospective cohort study. BMJ Open 2: e000825, 2012.

30. Qi P, Cheng SQ, Wang H, Li N, Chen YF and Gao CF: Serum microRNAs as biomarkers for hepatocellular carcinoma in Chinese patients with chronic hepatitis B virus infection. PLoS One 6: e28486, 2011.
31. Yan C, Zhang W, Shi X, Zheng J, Jin X and Huo J: MiR-760 suppresses non-small cell lung cancer proliferation and metastasis by targeting ROS1. Environ Sci Pollut Res Int 25: 18385-18391, 2018.

32. Cao L, Liu Y, Wang D, Huang L, Li F, Liu J, Zhang C, Shen Z, Gao Q, Yuan W and Zhang Y: MiR-760 suppresses human colorectal cancer growth by targeting BATF3/AP-1/cyclinD1 signaling. J Exp Clin Cancer Res 37: 83, 2018.

(i) $(-)$ This work is licensed under a Creative Commons Attribution-NonCommercial-NoDerivatives 4.0 International (CC BY-NC-ND 4.0) License. 